\title{
Cuidados de enfermería en una paciente con cáncer vulvar
}

Hernández-Estrada, Karen Melissa'; Hernández-Martínez, Nora²; Cruz-Chávez, Norma Edith³;

Cárdenas-Villarreal, Velia Margarita ${ }^{4}$

\section{RESUMEN}

Introducción: El carcinoma espinocelular o de células escamosas se presenta en órganos revestidos por epitelio escamoso como la piel, vagina y cuello uterino. El personal de enfermería juega un papel fundamental en el tratamiento de la persona que lo padece, para lograr mejorar su calidad de vida durante y posterior al tratamiento de la enfermedad. Objetivo: Exponer el cuidado de enfermería brindado a una paciente con cáncer de vulva durante la consulta oncológica en un hospital público en ciudad de Monterrey, Nuevo León; México durante 6 meses. Metodología: Estudio de caso, se fundamentó en las cinco etapas del proceso de atención de enfermería. Se utilizó la taxonomía de North American Nursing Diagnosis Association (NANDA), Nursing Intervention Classification (NIC) y Nursing Outcomes Classification (NOC) para fundamentar las intervenciones de enfermería realizadas. Presentación del caso: Como resultado de la valoración se obtuvo como diagnósticos de enfermería prioritarios: dolor agudo, estreñimiento, náuseas, desconocimiento del tratamiento y duelo por pérdida de un ser querido. Las intervenciones aplicadas fueron: Manejo del dolor, apoyo espiritual, educación sobre la enfermedad y manejo de la efectos secundarios de quimioterapia, radioterapia. Conclusiones: La paciente desarrollo estrategias no farmacológicas de manejo de dolor lo que ayudo al control del mismo, aumentó conocimientos sobre la enfermedad y sobre efectos del tratamiento, lo que favoreció a manejo de efectos secundarios de la radiación y quimioterapia, y afrontó el duelo por la pérdida del ser querido.

Palabras clave: Carcinoma; manejo del dolor; atención ambulatoria; enfermeros (DeCS; BIREME).

${ }^{1}$ Licenciada en Enfermería. Facultad de Enfermería, Universidad Autónoma de Nuevo León.

2. Doctora en Ciencias de Enfermería. Facultad de Enfermería, Universidad Autónoma de Nuevo León. E-mail: tevet7@hotmail.com

${ }^{3}$ Doctora en Ciencias de Enfermería. Facultad de Enfermería, Universidad Autónoma de Nuevo León.

${ }^{4}$ Doctora en Ciencias de Enfermería. Facultad de Enfermería, Universidad Autónoma de Nuevo León.

Recibido: $19 / 12 / 2018$

Aceptado: 22/03/2019

*Autor para correspondencia

\section{Cómo citar este artículo}

Hernández-Estrada KM, Hernández-Martínez N, Cruz-Chávez NE, Cárdenas-Villarreal VM. Cuidados de enfermería en una paciente con cáncer vulvar. SANUS. 2019; (9): 38-60. [Acceso____ ]; Disponible en: 


\section{Cuidados de enfermagem em patient com câncer vulvar}

\section{RESUMO}

Introdução: O carcinoma espinocelular ou células escamosas é comum em órgãos revestidos por epitélio escamoso como a pele, a vagina e o colo do útero. O pessoal de enfermagem tem um papel crítico no tratamento da pessoa com esta doença, a fim de melhorar sua qualidade de vida durante e após o tratamento da doença. Objetivo: Present the nursing care provided to a patient with vulvar cancer during the oncology consultation in a public hospital in the city of Monterrey, Nuevo León, México, during 6 months. Methodology: Estudo de caso baseado nas cinco etapas do processo de cuidar em enfermagem. A taxonomia da North American Nursing Diagnosis Association(NANDA), Nursing Intervention Classification (NIC), and Nursing Outcomes Classification (NOC) foram utilizados para fundamentar as intervenções de enfermagem realizadas.

Apresentação do caso: As a result of the assessment, o seguinte foi obtido como diagnóstico de enfermagem prioritário: dor aguda, constipação, náusea, ignorância de tratamento e luto devido à perda de um ente querido. As intervenções aplicadas foram: Manejo da dor, apoio espiritual, educação sobre a doença e manejo dos efeitos colaterais da quimioterapia e radioterapia. Conclusões: O paciente desenvolveu estratégias não farmacológicas para o controle da dor, que ajudou a controlá-lo, maior conhecimento sobre a doença e efeitos do tratamento, que favoreceu o manejo dos efeitos secundários da radiação e quimioterapia, e lidou com a dor devido à perda do ente querido.

Palavras chave: Carcinoma; manejo da dor; atendimento ambulatorial; enfermeiros (DeCS; BIREME)

\section{INTRODUCCIÓN}

El carcinoma espinocelular o de células escamosas (CEC) es un tipo de cáncer que se presenta en vagina y cuello uterino representa aproximadamente del 3 al $5 \%$ de los cánceres ginecológicos ${ }^{(1-3)}$. Se ha identificado que el diagnóstico y tratamiento del CEC provoca una experiencia inesperada, traumática y con severos efectos secundarios al tratamiento que impactan física y psicosocialmente al paciente y familia. La Guía de práctica clínica de diagnóstico y tratamiento de cáncer vaginal, refiere que el tratamiento del CEC debe de ser multidisciplinario, sin embargo, el personal de enfermería juega un papel fundamental para lograr que la persona pueda adaptarse a los tratamientos dolorosos, largos, agresivos, desfigurativos de la cirugía, quimioterapia y radioterapia. Así como ayudar a recuperar el funcionamiento diario para conseguir una calidad de vida durante y posterior al tratamiento de la enfermedad (4).

Es fundamental que el personal de enfermería del área clínica aplique la práctica basada en la evidencia, lo que facilite tomar decisiones que resulten apropiadas para intervenir en circunstancias clínicas específicas, como es en mujeres que padecen CEC. Una forma de utilizar y evaluar la práctica basada en evidencia es llevar a cabo estudios de caso, los cuales son una herramienta de investigación y una técnica de aprendizaje para el personal de enfermería, que sin duda contribuye al acercamiento entre la teoría, la práctica clínica y el paciente, con la finalidad de lograr un cuidado de salud con seguridad y responsabilidad $(4,5)$.

El objetivo del presente estudio es presentar la propuesta de cuidado efectuada a una mujer de mediana edad con un diagnóstico de cáncer vulvar, atendida en la consulta oncológica de un hospital público de tercer nivel desde su ingreso hasta seis meses que es dada de alta del tratamiento oncológico.

\section{METODOLOGÍA}

El caso se desarrolló en un centro de atención especializado de oncología ambulatoria ubicado en el norte de México durante el periodo de cinco meses, el estudio de caso, se fundamentó en las cinco etapas del proceso de enfermería( ${ }^{(6)}$. Para la valoración clínica se utilizó el instrumento de patrones funcionales de salud de Marjory Gordon ${ }^{(7)}$. Los enunciados del diagnóstico de enfermería, resultados esperados e intervenciones de enfermería se formularon con la interrelación de diagnósticos North American Nursing Diagnosis Association (NANDA), Nursing Outcomes Classification (NOC), Nursing Interventions Classificatión (NIC) ${ }^{(6-9)}$. Además se utilizó literatura relacionada y guías de práctica clínica relacionadas CEC y tratamientos alternos para el manejo del dolor, con el fin de sustentar a mayor 
profundidad las intervenciones a realizar. Para implementar el estudio de caso se le solicito previa autorización a la paciente y familia, se cuidaron todos los aspectos de privacidad y anonimato. A continuación, se presenta el estudio de caso desarrollado.

\section{PRESENTACIÓN DEL CASO}

\section{Antecedentes de ingreso}

Ingresa al servicio de consulta de oncología paciente femenina MLPA de 55 años (se le denominara como "PF"), soltera con una escolaridad de nivel medio superior, originaria de un estado del norte de México. El diagnóstico médico reportado fue CEC y se le practicó cirugía (vulvectomía) hace dos meses. Acude a consulta a recibir el cuarto ciclo de quimioterapia (4-4 sesiones) y radioterapia (7 sesiones). Antecedentes personales, niega presentar enfermedades crónicas, en relación con los antecedentes heredofamiliares, el padre fallece por hipertensión y la madre por diabetes, niega antecedentes familiares de cáncer, en abuelos maternos y paternos, padres y hermanos. Durante la valoración de enfermería, la entrevista fue realizada directamente con la paciente.

\section{Valoración y documentación por patrones funcionales de salud de Margory Gordon}

\section{Patrón 1. Percepción y manejo de la salud}

La "PF" reportó ser una persona sana durante toda su vida, comer saludable, aplicarse las vacunas que le corresponde a su edad y negó toxicomanías. Actualmente se encuentra orientada en tiempo, lugar y persona, con buena higiene física y dental. En cuanto a la percepción del estado de salud actual, la califica como mala salud. Ingiere paracetamol 500 mgs. por razón necesaria para el manejo del dolor, desconoce cuidados sobre su enfermedad y tratamientos a seguir.

\section{Patrón 2. Nutricional- metabólico}

La "PF" ha cambiado el patrón de nutrición desde que inicio el problema de salud actual, por indicación médica suspendió harinas, grasas, lácteos y picantes. Las últimas dos semanas presentó boca seca, sabor metálico, náuseas y vómito (promedio dos veces al día), "ella considera que es consecuencia del tratamiento actual que recibe". Solo ingiere en promedio medio litro de agua al día pérdida del apetito y bajar dos kilos de peso corporal en dos semanas. Se observó demacrada y cansada, la piel es pálida y reseca en todo su cuerpo, presenta hiperpigmentación periorbitaria (ojeras), se aprecia de más edad. En exámenes de laboratorio muestran neutrófilos $9.1 \times 10^{9} / \mathrm{L}$, hemoglobina $11.8 \mathrm{gr} / \mathrm{dl}$ y plaquetas
313,000 $\mu$ l. En parámetros bajos de hemoglobina y las plaquetas en parámetros normales. Actualmente reporta un Índice de Masa Corporal (IMC) de 20.7 kg/m2, normal para su edad

\section{Patrón 3. Eliminación}

El patrón de micción en promedio es de seis veces al día, de cantidad moderada, de color amarillo claro, olor característico sin ardor al orinar y sin salida involuntaria al esfuerzo. El patrón de eliminación intestinal es una vez cada tres días con dolor al evacuar, dolor abdominal y flatulencia frecuente. De acuerdo a la Escala de Heces de Bristol, la paciente se califica en tipo 2 ligero estreñimiento. El reporte de laboratorio del Examen General de Orina mostró datos normales de densidad, glucosa, pH, sangre y leucocitos. En este momento se considera con riesgo el patrón intestinal.

\section{Patrón 4. Actividad ejercicio}

La "PF" refiere no realizar labores del hogar por excesivo cansancio y fatiga. Con la valoración de la Escala Funcional de Capacidades de Autocuidado, se obtuvo un puntaje de 3, justificada por pasar la mayor parte del tiempo acostada, caminar con uso de andador, facies de dolor al caminar. Tiene instalado un catéter periférico corto número 24 en mano izquierda, de acuerdo a la valoración de la Escala de Flebitis es adecuado su cuidado y permanencia. Tensión arterial de 110/60 mmHg, llenado capilar de menos de dos segundos, pulso de 72 latidos por minuto, con frecuencia respiratoria de 22 por minuto, actualmente con síntomas de cansancio al caminar.

\section{Patrón 5. Sueño-descanso}

Durante la noche "PF" duerme en promedio seis horas y durante el día tres horas, aun así no se siente descansada. Bosteza repetidamente. Señala que el recordar a su madre que murió le interfiere dormir continuamente, asi como el dolor que presenta actualmente.

\section{Patrón 6. Cognitivo-perceptual}

Actualmente está orientada en tiempo, sabe leer y escribir. Señala la presencia de dolor muy fuerte localizado en la vulva, que se irradia hacia la espalda y recto, evaluado con la Escala Visual Análoga [EVA] 0 -10 lo califica con un 7. Reporta cefalea y dolor abdominal, el cual mejora con medicamento analgésico paracetamol cada 12 horas pero al pasar el efecto, éste regresa con una intensidad mayor o igual a 7 (escala EVA). En casa, le gustaría recibir información sobre su tratamiento actual. Usa lentes desde los 17 años y se le diagnóstico hipoacusia recientemente en ambos oídos. La necesidad de conocer más sobre el proceso de la enfermedad y su cuidado es de interés para la paciente. 


\section{Patrón 7. Autopercepción- Auto concepto}

La PF reportó la muerte de su madre hace un año, la cual vivía con ella, en estos momentos refiere sentirse triste, enferma y sola. Su proyecto de vida cambio totalmente, ya que planeaba trabajar, actualmente por la enfermedad que padece y su tratamiento, vive con su única hermana y depende totalmente de ella. Se percibe con confianza de salir adelante de su enfermedad por el tratamiento médico que está recibiendo actualmente.

\section{Patrón 8. Rol-Relaciones}

El rol social de la "PF" es de hermana y paciente, tiene comunicación con su familia (hermana, cuñado y sobrina), además, de tener con ellos una red de apoyo emocional y económico para afrontar su enfermedad. Así mismo refirió buena relación con vecinos y con el personal de enfermería y equipo de salud de la institución donde recibe el tratamiento. Durante la consulta de oncología saluda y platica con las enfermeras y las pacientes.

\section{Patrón 9. Sexualidad reproducción}

La "PF" niega vida sexual activa, la fecha de su última menstruación fue hace seis años. Nunca se realizó un examen de detección de cáncer de mama y cervico-vaginal ya que no lo consideraba necesario debido a que nunca tuvo relaciones sexuales. Se le practico cirugía (vulvectomía) hace dos meses. Actualmente presenta escasa salida de secreción amarillenta por vagina la cual no es fétida sin riesgo para su vida.

\section{Patrón 10 Afrontamiento tolerancia al estrés}

La"PF" refiere casi nunca enojarse, presentar estrés diario por no poder realizar sus actividades diarias, debido al cansancio que ha manifestado desde que inicio el tratamiento de quimioterapia. El estrés lo disminuye estando a solas y pensando positivo. Durante el tratamiento de quimioterapia se muestra tranquila, y refiere confianza por el personal de enfermería.

\section{Patrón 11. Valores y creencias}

Practica la religión católica, devota de la virgen María, Dios y San Judas no ha acudido a su iglesia desde que le practicaron la cirugía. La oración le da tranquilidad y paz. Tiene mucha fe que saldrá adelante. Menciona que para ella primero es Dios y su escala de valores la clasifica en primer lugar el amor, la responsabilidad, honestidad y último la solidaridad. Niega conocer los programas de salud dirigidos a la mujer, no lo consideró importante debido a no tener una vida sexual activa ni embarazos. Este patrón se considera disfuncional.

\section{Identificación de necesidades de cuidado}

Se formularon diagnósticos de enfermería (reales, de riesgo y promoción de la salud) extraídos de la NANDA (Tabla 1), se validaron y se priorizaron en base a las necesidades sentidas de la paciente.

\section{Validación de diagnósticos con la paciente}

La "PF" señalo que los diagnósticos de enfermería prioritarios que consideraba debían de resolverse primero eran: 00132 Dolor agudo, el 00136 Duelo por muerte de una persona significativa (madre), 00161 Disposición para mejorar los conocimientos sobre la enfermedad y tratamiento de quimioterapia y radioterapia.

\section{Fundamentación del diagnóstico de Enfermería}

El dolor considerado como una experiencia sensorial y emocional no agradable, es el síntoma principal en el $40 \%$ de los pacientes oncológicos en tratamiento y con una enfermedad avanzada. Por lo común, el tratamiento del dolor se limita a resolver solo las respuestas físicas con fármacos, sin considerar las respuestas emocionales y espirituales que sueles estar relacionadas con el dolor y que pudieran ser resueltas integralmente con tratamientos no farmacológicos por un equipo multiprofesional(10).

Cuando al dolor físico se agrega el dolor por la pérdida de un ser querido como en este caso de la "PF", el duelo puede persistir por mucho más tiempo, si no se le ayuda a afrontar el proceso de lidiar emocionalmente con la pérdida. Una persona con cáncer puede ser emocionalmente más lábil al afrontar una pérdida, lo cual puede reflejar más pena, tristeza y cambios a nivel emocional (11). Una aflicción normal se refleja con la aceptación de la pérdida del ser querido y seguir adelante de una forma saludable. Conjugar terapia farmacológica y no farmacológica es prioritario para mejorar la calidad de vida de los pacientes con dolor y problemas emocionales. Se ha evidenciado que el masaje facial (reflexología) es un cuidado paliativo no farmacológico que puede tener beneficios en las personas con cáncer, ya que aumenta la relajación, el sueño, reduce el dolor y los síntomas psicológicos, como la depresión y ansiedad. Así mismo aumenta la liberación de endorfinas y potencia los efectos terapéuticos de los analgésicos ${ }^{(12,13)}$.

La mayoría de los pacientes que padecen cáncer les produce incertidumbre o estrés por el tratamiento que recibirán. La habilidad de la enfermera podría disminuirlos, en la medida que provea información periódica y cierta sobre su estado de salud al paciente y a su familia. Es trascendental que la enfermera identifique situaciones de riesgo por falta de información sobre la enfermedad y tratamientos a realizar, para trabajar en equipo un plan individual con el paciente. El afrontamiento de la enfermedad permite al paciente oncológico aceptar, manejar y sobreponerse a su proceso salud/ enfermedad, y a la vez a mejorar la calidad de vida ${ }^{(13)}$. 
Tabla 1. Diagnósticos de enfermería NANDA.

\begin{tabular}{|c|c|c|}
\hline $\begin{array}{c}\text { Etiqueta } \\
\text { Diagnóstica }\end{array}$ & $\begin{array}{c}\text { Factores Relacionados } \\
\text { u de riesgo }\end{array}$ & $\begin{array}{l}\text { Características definitorias } \\
\text { (Manifestaciones clínicas) }\end{array}$ \\
\hline $\begin{array}{l}\text { DOMINIO 12: Confort } \\
\text { CLASE 1: Confort físico } \\
00132 \text { Dolor agudo }\end{array}$ & $\begin{array}{l}\text { Lesión por agente biológico (cirugía previa por } \\
\text { tumor en vulva, dolor que irradia hacia espalda, } \\
\text { cefalea) }\end{array}$ & $\begin{array}{l}\text { Auto informe de intensidad con } \\
\text { escala estandarizada de dolor, } \\
\text { cambios en el apetito, conducta } \\
\text { expresiva, conducta protectora, } \\
\text { expresión facial de dolor, postura } \\
\text { para aliviar el dolor } \\
\text { (dolor continuo que se irradia a } \\
\text { la espalda, realiza de } 3 \text { a } 5 \text { comi- } \\
\text { das en muy poca cantidad, escala } \\
\text { EVA de } 7 \text {, facies de dolor) }\end{array}$ \\
\hline $\begin{array}{l}\text { DOMINIO9: Afronta- } \\
\text { miento/ tolerancia al } \\
\text { estrés } \\
\text { CLASE 2: Respuestas de } \\
\text { afrontamiento } \\
\text { 00136 Duelo }\end{array}$ & $\begin{array}{l}\text { Muerte de una persona significativa (muerte de } \\
\text { madre, cambio de residencia) }\end{array}$ & $\begin{array}{l}\text { Alteración del nivel de actividad, } \\
\text { conexión frecuente con la perso- } \\
\text { na fallecida } \\
\text { (refiere sentirse triste por la muer- } \\
\text { te de su madre, extraña su casa } \\
\text { donde vivía). }\end{array}$ \\
\hline $\begin{array}{l}\text { DOMINIO3: Eliminación e } \\
\text { intercambio } \\
\text { CLASE2: Función gas- } \\
\text { trointestinal } \\
00011 \text { Estreñimiento }\end{array}$ & $\begin{array}{l}\text { Farmacológicos: Agente farmacéutico. } \\
\text { Funcionales: Actividad física diaria promedio es } \\
\text { menor a la recomendada por sexo y edad Fisioló- } \\
\text { gicos: Ingesta insuficiente de líquidos. } \\
\text { (tratamiento de quimioterapia y radioterapia, } \\
\text { pasar acostada mayoría del tiempo, estrés por no } \\
\text { realizar actividad del hogar, cambio de alimenta- } \\
\text { ción) }\end{array}$ & $\begin{array}{l}\text { Dolor abdominal, flatulencia, } \\
\text { disminución de la frecuencia, } \\
\text { heces formadas y dolor al defecar, } \\
\text { cefalea, vómito. } \\
\text { (Dolor abdominal, flatulencia, } \\
\text { disminución de la frecuencia para } \\
\text { evacuar, escala Bristol tipo 2, } \\
\text { peristaltismo disminuido, cefalea, } \\
\text { vómito). }\end{array}$ \\
\hline $\begin{array}{l}\text { DOMINIO 12: Confort } \\
\text { CLASE 1: Confort físico } \\
\text { Náuseas }\end{array}$ & $\begin{array}{l}\text { Situacionales: Sabores desagradables; biofísicos: } \\
\text { régimen del tratamiento (inicio de quimioterapia y } \\
\text { radioterapia). }\end{array}$ & $\begin{array}{l}\text { Aumento de la salivación, náu- } \\
\text { seas, sabor metálico, aversión a } \\
\text { los alimentos } \\
\text { (realiza de } 3 \text { a } 5 \text { comidas en muy } \\
\text { poca cantidad, cambio de alimen- } \\
\text { tación, náuseas y vómitos, con- } \\
\text { sume menos de } 1 \text { litro de agua, } \\
\text { sabor metálico en la boca). }\end{array}$ \\
\hline $\begin{array}{l}\text { DOMINIO 4: Actividad y } \\
\text { reposo } \\
\text { CLASE 5: Autocuidado } \\
00193 \text { Descuido personal }\end{array}$ & $\begin{array}{l}\text { Abuso de sustancias (tratamiento de quimiotera- } \\
\text { pia, uso de citotóxico, refiere sentirse triste, muer- } \\
\text { te de un ser querido, se estresa por no realizar } \\
\text { actividades) }\end{array}$ & $\begin{array}{l}\text { No adherencia a las actividades } \\
\text { relacionadas con la salud } \\
\text { (desconocía realizar exámenes de } \\
\text { detección oportuna de cáncer de } \\
\text { mama y cérvix) }\end{array}$ \\
\hline
\end{tabular}




\begin{tabular}{|c|c|c|}
\hline $\begin{array}{l}\text { DOMINIO } 2 \text { Nutrición } \\
\text { CLASE 1: ingestión } \\
00002 \text { Desequilibrio nu- } \\
\text { tricional ingesta inferior a } \\
\text { las necesidades }\end{array}$ & $\begin{array}{l}\text { Ingesta insuficiente de alimentos (dejo las hari- } \\
\text { nas, grasa, lácteos y picantes), factores biológicos } \\
\text { Tumoración cancerosa en región vulvar. }\end{array}$ & $\begin{array}{l}\text { Palidez de mucosas oral. Dolor } \\
\text { abdominal, alteración del senti- } \\
\text { do del gusto, informe de ingesta } \\
\text { inferior a las cantidades diarias } \\
\text { recomendadas, } \\
\text { Pérdida de peso (2 kilos en dos } \\
\text { semanas, Dolor abdominal, realiza } \\
\text { de } 3^{a} 5 \text { comidas en muy poca } \\
\text { cantidad, cambio de alimentación, } \\
\text { consume menos de } 1 \text { litro de } \\
\text { agua, sabor metálico en la boca) }\end{array}$ \\
\hline $\begin{array}{l}\text { DOMINIO } 2 \text { nutrición } \\
\text { CLASE 5: hidratación } \\
00027 \\
\text { Déficit del volumen de } \\
\text { líquidos }\end{array}$ & $\begin{array}{l}\text { Pérdida importante del volumen de líquidos (vo- } \\
\text { mito } 2 \text { veces o más al día). }\end{array}$ & $\begin{array}{l}\text { Alteración de la turgencia de la } \\
\text { piel; disminución de la turgencia } \\
\text { de la lengua, sequedad de las } \\
\text { mucosas, perdida súbita de peso } \\
\text { (2 kilos en dos semanas) vómitos. } \\
\text { (consume menos de } 1 \text { litro de } \\
\text { agua, náuseas y vómitos, piel } \\
\text { reseca) }\end{array}$ \\
\hline $\begin{array}{l}\text { DOMINIO 4: Actividad y } \\
\text { reposo } \\
\text { CLASE 2: Actividad y } \\
\text { ejercicio } \\
00088 \\
\text { Deterioro de la ambula- } \\
\text { ción }\end{array}$ & $\begin{array}{l}\text { Deterioro de la visión, mala condición física, } \\
\text { fuerza muscular insuficiente, dolor (uso de lentes } \\
\text { desde los } 17 \text { años, hipoacusia, dolor, cefalea). }\end{array}$ & $\begin{array}{l}\text { Disminución de la habilidad para } \\
\text { caminar distancias requeridas. } \\
\\
\text { (uso de andador, permanece } \\
\text { acostada la mayor parte de tiem- } \\
\text { po, la escala funcional de capa- } \\
\text { cidad de autocuidado presenta } \\
\text { puntaje 3, cansancio y dolor) }\end{array}$ \\
\hline $\begin{array}{l}\text { DOMINIO } 11 \\
\text { Seguridad y protección } \\
\text { CLASE 2: Lesión física } \\
00155 \text { Riesgo de caída }\end{array}$ & $\begin{array}{l}\text { Fisiológicos: deterioro de la movilidad, neoplasia, } \\
\text { disminución de la fuerza en extremidades inferio- } \\
\text { res, Edad: Uso de dispositivos de ayuda, Medica- } \\
\text { ción: agente farmacéutico, dificultades visuales, } \\
\text { dificultades auditivas (uso de lentes desde los } 17 \\
\text { años, hipoacusia, dolor, cefalea, uso de andador, } \\
\text { baja energía). }\end{array}$ & \\
\hline $\begin{array}{l}\text { DOMINIO5: Percepción/ } \\
\text { cognición } \\
\text { CLASE 4: Cognición } \\
00161 \text { Disposición para } \\
\text { mejorar los conocimien- } \\
\text { tos }\end{array}$ & & $\begin{array}{l}\text { Expresa el deseo de mejorar en el } \\
\text { aprendizaje de su tratamiento. } \\
\text { (le gustaría recibir información de } \\
\text { la quimioterapia y radioterapia) }\end{array}$ \\
\hline
\end{tabular}

Fuente: $\mathrm{NANDA}^{6}$ y Elaboración propia 
Tabla 2. Plan de Intervención / plan de cuidados

\begin{tabular}{|c|c|c|c|}
\hline $\begin{array}{c}\text { Problema/ } \\
\text { Diagnóstico de en- } \\
\text { fermería NANDA }\end{array}$ & $\begin{array}{l}\text { Resultado esperado } \\
\text { (NOC) }\end{array}$ & $\begin{array}{l}\text { Objetivos plantea- } \\
\text { dos }\end{array}$ & Intervención/NIC \\
\hline $\begin{array}{l}\text { Dominio 12: Confort. } \\
\text { Clase 1: Confort } \\
\text { físico. } \\
\text { Código 00132: Dolor } \\
\text { agudo, relacionado } \\
\text { con lesión por agen- } \\
\text { te biológico manifes- } \\
\text { tada por Auto infor- } \\
\text { me de intensidad con } \\
\text { escala estandarizada } \\
\text { de dolor, cambios en } \\
\text { el apetito, conducta } \\
\text { expresiva, conducta } \\
\text { protectora, expre- } \\
\text { sión facial de dolor, } \\
\text { postura para aliviar el } \\
\text { dolor }\end{array}$ & $\begin{array}{l}\text { Dominio: Conocimiento } \\
\text { y conducta de salud } \\
\text { (IV). } \\
\text { Clase Q: Conducta de } \\
\text { salud, } \\
\text { Control del dolor (1605) } \\
\text { Acciones personales } \\
\text { para control del dolor. } \\
\text { Escala de medición: } \\
\text { Nunca demostrado } \\
\text { hasta } \\
\text { Siempre demostrado } \\
\text { Evaluación de inicio: } \\
\text { puntuación Diana } 1 \\
\text { Mantener } 1 \\
\text { Aumentar } 4\end{array}$ & $\begin{array}{l}\text { Aplicar el masaje fa- } \\
\text { cial ( } 30 \text { min) durante } \\
\text { la administración } \\
\text { de la quimioterapia } \\
\text { (4 sesiones una vez } \\
\text { a la semana) para } \\
\text { disminuir el dolor. } \\
\\
\text { Disminuir el dolor al } \\
\text { administrar medica- } \\
\text { mento (analgésico) } \\
\text { con horario prescri- } \\
\text { to para evitar picos } \\
\text { de dolor intenso. }\end{array}$ & $\begin{array}{l}\text { Campo } 1 \text { Fisiológico básico. } \\
\text { Clase E: Fomento a la comodidad Física. } \\
\text { Intervención 1400: Manejo del dolor. } \\
\text { Alivio del dolor, Disminución del dolor a } \\
\text { un nivel de tolerancia que sea aceptado } \\
\text { para el paciente. } \\
\text { Actividades: } \\
\text { Realizar una valoración del dolor en } \\
\text { la paciente que incluya la localización, } \\
\text { duración, intensidad y factores desenca- } \\
\text { denantes en el área de quimioterapia con } \\
\text { evaluación de la escala de EVA. } \\
\text { Aplicar el uso de técnicas no farmacoló- } \\
\text { gicas (masaje facial) en la paciente para } \\
\text { que facilitar el alivio del dolor durante la } \\
\text { aplicación del tratamiento de quimiotera- } \\
\text { pia en el área de quimioterapia. } \\
\text { Corroborar que la paciente reciba los } \\
\text { analgésicos antes de la quimioterapia } \\
\text { paracetamol } 500 \text { mgs vía oral. }\end{array}$ \\
\hline
\end{tabular}

Continúa...

\section{Desarrollo del plan de intervención /cuidados}

En base a las necesidades de la paciente se desarrolló el plan de cuidados. En la tabla 2 se muestra la relación de los diagnósticos de enfermería, resultado esperado, objetivos planteados e intervenciones NIC realizadas.

En la tabla 3, se muestran los resultados obtenidos de la evaluación control del dolor (1605), al inicio y final de la intervención. En ella se observa que la paciente mejoró sustancialmente en todos los indicadores. De acuerdo a la escala NOC, la paciente reportó una puntuación Diana de 1, posterior a la intervención aumento a 4. Se le habilitó para realizarse masaje facial como método no farmacológico durante su tratamiento de quimioterapia y además se le enseño como hacerlo en su hogar, continuo con los analgésicos indicados por su médico.

Con respecto al duelo que presentaba la paciente también se lograron cambios en su evaluación, como lo muestra la tabla 4. La paciente logró abrir sus sentimientos sobre la pérdida de su madre, además busco apoyo social, se integró al grupo de apoyo de la Cruz Rosa y apoyo espiritual (asistió a la capilla del hospital, se le entrego un rosario y libro de oración). De acuerdo a la escala NOC, la paciente se evalúo con una puntuación Diana de inicio de 1 y al final de la intervención en 5 .

En relación con los indicadores obtenidos de la evaluación del conocimiento manejo del cáncer vulvar, al inicio de la intervención la PF reportó 1 en la mayoría de los indicadores a evaluar, al final de la intervención la paciente aumento su conocimiento a sustancial y extenso quedando su evaluación final en 4 y 5 como se observa en la tabla 5.

\section{CONCLUSIONES}

En este caso se identificó una progresión en los resultados. Durante el tiempo de desarrollo del caso se lograron cambios significativos en el control del dolor, manejo del duelo por la muerte de su familiar y aumento de conocimiento sobre el tratamiento del CEC mediante el uso de las taxonomías NANDA, NIC y NOC.

Por lo general, el dolor percibido en un paciente oncológico es identificado como un problema prioritario de salud dado que afecta la calidad de vida del paciente y la del cuidador 


\begin{tabular}{|c|c|c|c|}
\hline $\begin{array}{l}\text { Dominio 9: Afronta- } \\
\text { miento/ tolerancia al } \\
\text { estrés. } \\
\text { Clase 2: respuestas } \\
\text { de afrontamiento. } \\
\text { Código 00136: } \\
\text { Duelo relacionado } \\
\text { con Muerte de una } \\
\text { persona significativa } \\
\text { manifestado por Al- } \\
\text { teración del nivel de } \\
\text { actividad, manteni- } \\
\text { miento de la cone- } \\
\text { xión con la persona } \\
\text { fallecida }\end{array}$ & $\begin{array}{l}\text { Dominio III: salud psi- } \\
\text { cosocial. } \\
\text { Clase N: adaptación } \\
\text { psicosocial. } \\
\text { Código (1304): Resolu- } \\
\text { ción de la aflicción, ac- } \\
\text { ciones personales para } \\
\text { ajustar pensamientos, } \\
\text { sentimientos y conduc- } \\
\text { tas a la pérdida real o } \\
\text { inminente. } \\
\text { Escala de medición: } \\
\text { Nunca demostrado has- } \\
\text { ta siempre demostrado } \\
\text { Evaluación de inicio } \\
\text { puntuación Diana } 1 \\
\text { Mantener } 1 \\
\text { Aumentar } 5\end{array}$ & $\begin{array}{l}\text { 1.- Establecer una } \\
\text { comunicación } \\
\text { que desemboque } \\
\text { confianza entre } \\
\text { paciente enfermera } \\
\text { por medio de la } \\
\text { empatía que se lle- } \\
\text { vara a cabo durante } \\
\text { todo el proceso de } \\
\text { interacción con la } \\
\text { paciente en un pro- } \\
\text { ceso de } 3 \text { semanas } \\
\\
\text { 2. Incorporar a la } \\
\text { paciente a grupos } \\
\text { de apoyo dentro de } \\
\text { la institución con } \\
\text { el fin de que reciba } \\
\text { ayuda para superar } \\
\text { su perdida. } \\
\text { erdion } \\
\text { 3. Ayudar a la pa- } \\
\text { ciente a expresar sus } \\
\text { sentimientos y libe- } \\
\text { rarse por medio de } \\
\text { una plática de una } \\
\text { duración de } 60 \text { min } \\
\text { en la sala de espera } \\
\text { de radioterapia. } \\
\text { tución hospitalaria. } \\
\text { de apoyo, escucha y } \\
\text { integración a grupos } \\
\text { ciente a superar la } \\
\text { muerte de su madre } \\
\text { por medio de apoyo } \\
\text { espiritual mediante } \\
\text { actividades en rela- } \\
\text { ión a las creencias, } \\
\text { inti }\end{array}$ & $\begin{array}{l}\text { Campo 3: Conductual. } \\
\text { Clase R: ayudar a hacer frente a situacio- } \\
\text { nes difíciles. } \\
\text { Intervención } \\
\text { Código 5420: Apoyo espiritual. } \\
\text { Hacer sentir al paciente un equilibrio y } \\
\text { conexión con un poder sobrenatural. } \\
\text { Utilizar la comunicación con la paciente } \\
\text { para establecer confianza y empatía en } \\
\text { durante todo el proceso desde valoración } \\
\text { hasta cierre de proceso, por medio de } \\
\text { la utilización de patrones funcionales de } \\
\text { Gordon en las áreas en las que se tenga } \\
\text { contacto con la paciente. } \\
\text { Búsqueda de grupos de apoyo para que } \\
\text { la paciente escuche otros casos similares } \\
\text { y tenga otras perspectivas, integrarla en el } \\
\text { grupo "Cruz Rosa" que brindan apoyo en } \\
\text { el departamento de oncología a todas las } \\
\text { mujeres con cáncer antes de ingresar a su } \\
\text { tratamiento de radioterapia. } \\
\text { Escuchar los sentimientos de la paciente } \\
\text { para ayudarle a desahogarse y liberarse } \\
\text { de la tristeza durante el tratamiento de } \\
\text { quimioterapia en dicha área. } \\
\text { Apoyo espiritual al acompañar atenta- } \\
\text { mente a la paciente a rezar si así lo desea, } \\
\text { en la capilla del hospital después de la } \\
\text { aplicación de radioterapia. } \\
\text { Expresar simpatía con los sentimientos de } \\
\text { la paciente durante todo el proceso en el } \\
\text { cual se está teniendo contacto con ella. }\end{array}$ \\
\hline
\end{tabular}

primario. Con frecuencia el personal de salud valora solo unidimensionalmente (físico), el dolor olvidando que este afecta otras áreas de la vida como lo emocional, familiar, social, por lo que el profesional de enfermería debe dominar la aplicación de intervenciones farmacológicas y no farmacológicas y trabajar multidisciplinariamente.

Además, es importante que el personal de enfermería establezca vínculos afectivos, de confianza y de comunicación con el paciente y la familia para ayudar a enfrentar la enfermedad, los procedimientos y las complicaciones del tratamiento, transformándose en ejemplos de vida.

Un caso clínico es una estructura sistemática y lógica que permite transmitir el conocimiento, acercar la teoría con la práctica clínica aplicada en conjunto con los pacientes. Los casos clínicos permiten a los profesionales de enfermería conocer como aplican la evidencia y discutirlas con otros profesionales de enfermería para ayudar a tomar decisiones en el manejo de los pacientes a su cargo. El manejo de las taxonomías NANDA, NOC y NIC, promueven el análisis del proceso de cuidado de enfermería. 


\begin{tabular}{|c|c|c|c|}
\hline $\begin{array}{l}\text { Dominio 5: percep- } \\
\text { ción/cognición. } \\
\text { Clase 4: Cognición. } \\
\text { Código 00161: } \\
\text { Disposición para } \\
\text { mejorar los conoci- } \\
\text { mientos manifestado } \\
\text { por que Expresa el } \\
\text { deseo de mejorar en } \\
\text { el aprendizaje de su } \\
\text { tratamiento }\end{array}$ & $\begin{array}{l}\text { Dominio IV: Conoci- } \\
\text { miento y conducta de } \\
\text { salud. } \\
\text { Clase S: Conocimientos } \\
\text { sobre salud. } \\
\text { Escala de resultado: Co- } \\
\text { digo1833: Conocimien- } \\
\text { to: manejo del cáncer. } \\
\text { Grado de conocimiento } \\
\text { transmitido sobre el } \\
\text { cáncer, su tratamiento } \\
\text { y la prevención de la } \\
\text { progresión de la enfer- } \\
\text { medad y las complica- } \\
\text { ciones. } \\
\text { Escala de medición. } \\
\text { Ningún conocimiento } \\
\text { hasta conocimiento } \\
\text { extenso } \\
\text { Evaluación de inicio } \\
\text { puntuación Diana } 1 \\
\text { Mantener } 1 \\
\text { Aumentar } 5\end{array}$ & $\begin{array}{l}\text { Brindar conocimien- } \\
\text { tos a la paciente } \\
\text { sobre el cáncer en } \\
\text { relación al manejo } \\
\text { de la quimiotera- } \\
\text { pia, radioterapia y } \\
\text { los efectos de los } \\
\text { mismos mediante } \\
\text { educación en la } \\
\text { sala de espera con } \\
\text { apoyo de un tríptico } \\
\text { y desarrollo de pro- } \\
\text { cedimientos. }\end{array}$ & $\begin{array}{l}\text { Campo 4: Seguridad. } \\
\text { Clase V: Control de riesgos. } \\
\text { Intervención: } \\
\text { Código 6600: Manejo de la radioterapia } \\
\text { Ayudar al paciente a comprender y a } \\
\text { minimizar los efectos secundarios de un } \\
\text { tratamiento con radiación posterior a su } \\
\text { administración y en el hogar. } \\
\text { Proporcionar información a la pacien- } \\
\text { te respecto a la quimioterapia, efectos } \\
\text { secundarios y como tratar los mismos } \\
\text { posterior a su aplicación y en el hogar. } \\
\text { Verificar que en la sala de espera de } \\
\text { Radioterapia a base de una clase y apoyo } \\
\text { con tríptico de información. } \\
\text { el área de quimioterapia durante la aplica- } \\
\text { ción del tratamiento } \\
\text { durante o después y ponerlo en práctica } \\
\text { Campo 2: Fisiológico complejo } \\
\text { Clase H: Control de fármacos } \\
\text { Intervención } \\
\text { Código } 2240 \text { de: Manejo de la quimiote- } \\
\text { rapia } \\
\text { Orientar al paciente y a la familia sobre } \\
\text { los efectos secundarios de los agentes } \\
\text { antineoplásicos. } \\
\text { cisplatino (antineoplásico) para el trata- } \\
\text { miento de su enfermedad por medio de } \\
\text { enseñanza a base de una clase y tríptico } \\
\text { informativo en la sala de espera de qui- } \\
\text { mioterapia. }\end{array}$ \\
\hline
\end{tabular}

Fuente: $\mathrm{NANDA}^{6}, \mathrm{NIC}^{8}, \mathrm{NOC}^{9}$ y Elaboración propia. 
Tabla 3. Evaluación indicadores del Control del dolor

\begin{tabular}{|c|c|c|c|c|c|c|}
\hline \multicolumn{2}{|r|}{ Indicadores } & $\begin{array}{c}\text { Nunca } \\
\text { demostrado }\end{array}$ & $\begin{array}{l}\text { Raramente } \\
\text { demostrado }\end{array}$ & $\begin{array}{c}\text { A veces } \\
\text { demostrado }\end{array}$ & $\begin{array}{l}\text { Frecuentement } \\
\text { e demostrado }\end{array}$ & $\begin{array}{c}\text { Siempre } \\
\text { demostrado }\end{array}$ \\
\hline 160502 & Reconoce el comienzo del dolor & 1 & 2 & 0 & 4 & $\Delta \theta$ \\
\hline 160501 & Reconoce factores causales & 1 & 2 & 30 & 4 & $5 \Delta \theta$ \\
\hline 160503 & Utiliza medidas preventivas & 1 & 2 & 3 & 40 & $5 \Delta \theta$ \\
\hline 160504 & $\begin{array}{l}\text { Utiliza medidas de alivio no } \\
\text { analgésicas }\end{array}$ & ${ }_{1} \mathrm{O}$ & 2 & & 4 & $5 \Delta \theta$ \\
\hline 160505 & $\begin{array}{l}\text { Utiliza los analgésicos de forma } \\
\text { apropiada }\end{array}$ & 1 & 2 & 3 & 4 & ${ }_{5} \Delta \diamond$ \\
\hline 160513 & $\begin{array}{l}\text { Refiere cambios en los síntomas al } \\
\text { personal }\end{array}$ & ${ }_{1} \mathrm{O}$ & 2 & 3 & 4 & $5 \diamond \Delta$ \\
\hline 160507 & $\begin{array}{l}\text { Refiere síntomas incontrolables al } \\
\text { personal }\end{array}$ & ${ }_{1} 0$ & 2 & 3 & $4 \diamond$ & $5 \Delta$ \\
\hline 160508 & Utiliza los recursos disponibles & ${ }_{1} \mathrm{O}$ & 2 & 3 & $4 \diamond$ & $5 \Delta$ \\
\hline 160509 & $\begin{array}{l}\text { Reconoce síntomas asociados al } \\
\text { dolor }\end{array}$ & 10 & 2 & 3 & $4 \diamond$ & $5 \Delta$ \\
\hline 160511 & Refiere dolor controlado & 10 & 2 & 3 & 4 & $5 \Delta \bullet$ \\
\hline
\end{tabular}

Fuente: $\operatorname{NOC}^{9}$ y Elaboración propia como se encontro 0 objetivo a lograr $\Delta$ logro $\diamond$

Tabla 4. Evaluación Indicadores del Resolución de la Aflicción

\begin{tabular}{|c|c|c|c|c|c|c|}
\hline \multicolumn{2}{|r|}{ Indicadores } & \multirow{2}{*}{$\begin{array}{c}\begin{array}{c}\text { Nunca } \\
\text { demostrado }\end{array} \\
1\end{array}$} & \multirow{2}{*}{$\begin{array}{c}\begin{array}{c}\text { Frecuentemente } \\
\text { demostrado }\end{array} \\
2\end{array}$} & \multirow{2}{*}{$\begin{array}{c}\text { A veces } \\
\text { demostrado }\end{array}$} & \multirow{2}{*}{$\begin{array}{c}\begin{array}{c}\text { Frecuentemente } \\
\text { demostrado }\end{array} \\
{ }_{4} \mathrm{O}\end{array}$} & \multirow{2}{*}{$\begin{array}{c}\begin{array}{c}\text { Siempre } \\
\text { demostrado }\end{array} \\
\Delta_{5} \diamond\end{array}$} \\
\hline 130401 & Expresa sentimientos sobre la perdida & & & & & \\
\hline 130402 & $\begin{array}{l}\text { Expresa creencias espirituales sobre la } \\
\text { muerte }\end{array}$ & 1 & 2 & 3 & 4 & $\theta$ \\
\hline 130403 & Verbaliza la realidad de la perdida & 1 & ${ }_{2} \mathrm{C}$ & 3 & 4 & $\Delta_{5} \diamond$ \\
\hline 130404 & Verbaliza la aceptación de la perdida & 1 & 2 & 3 & 4 & $\Delta_{5} \boldsymbol{\theta}$ \\
\hline 130405 & Describe el significado de la perdida & 1 & 2 & 3 & 4 & $\Delta_{5} \diamond$ \\
\hline 130411 & $\begin{array}{l}\text { Refiere disminución de la preocupación con } \\
\text { la perdida }\end{array}$ & 1 & 2 & 3 & 4 & $\Delta_{5} \boldsymbol{\theta}$ \\
\hline 130412 & Mantiene el ambiente de vida & 1 & 2 & ${ }_{3} \mathrm{O}$ & 4 & $5 \diamond \Delta$ \\
\hline 130413 & Mantiene el cuidado e higiene personales & 1 & 2 & 3 & 4 & $\Delta \Delta$ \\
\hline 130414 & Refiere sueño adecuado & 1 & 2 & 3 & 4 & $5 \diamond \Delta$ \\
\hline 1300415 & Refiere una ingestión nutricional adecuada & 1 & ${ }_{2}$ & 3 & 48 & ${ }_{5} \Delta$ \\
\hline 130417 & Busca apoyo social & ${ }_{1}$ & 2 & 3 & 48 & ${ }_{5} \Delta$ \\
\hline 130418 & $\begin{array}{l}\text { Comparte la perdida con otros seres } \\
\text { queridos }\end{array}$ & ${ }_{1}($ & 2 & 3 & 4 & $5 \diamond \Delta$ \\
\hline 130419 & Refiere implicación de actividades sociales & ${ }_{1}$ & 2 & 3 & 4 & $5 \diamond \Delta$ \\
\hline 130420 & Progresa a lo largo de las fases de aflicción & 1 & 2 & 3 & $4 \diamond \Delta$ & 5 \\
\hline 130421 & Expresa expectativas positivas sobre el futuro & 1 & 2 & 3 & ${ }_{4} \mathrm{O}$ & $5 \diamond \Delta$ \\
\hline
\end{tabular}

Fuente: $\operatorname{NOC}^{9}$ y Elaboración propia como se encontro $O$ objetivo a lograr $\Delta$ logro $\diamond$ 
Tabla 5. Evaluación Indicadores de Conocimiento: manejo del cáncer vulvar

\begin{tabular}{|c|c|c|c|c|c|c|}
\hline & Indicadores & $\begin{array}{c}\text { Ningutn } \\
\text { conocimiento }\end{array}$ & $\begin{array}{l}\text { Coneciminto } \\
\text { scase }\end{array}$ & $\begin{array}{l}\text { Conocimiento } \\
\text { maderude }\end{array}$ & $\begin{array}{l}\text { Candiminto } \\
\text { sustantial }\end{array}$ & $\begin{array}{l}\text { Conocimisnto } \\
\text { antense }\end{array}$ \\
\hline 183102 & $\begin{array}{l}\text { Synar y ginomin dell } \\
\text { anner }\end{array}$ & 1 & 2 & 3 & $\begin{array}{l}4 \\
6\end{array}$ & \\
\hline 183303 & 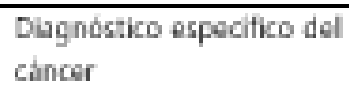 & 1 & 20 & 3 & $4 \phi$ & $5 \Delta$ \\
\hline 183364 & 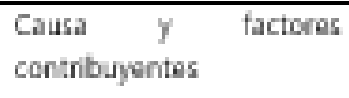 & 1 & 2 & 3 & 4 & $\begin{array}{l}5 \\
9 A\end{array}$ \\
\hline 18336 & $\begin{array}{l}\text { Opclones twapesuticas } \\
\text { dteponibias }\end{array}$ & 0 & 2 & 3 & 40 & $\begin{array}{l}5 \\
\mathrm{~A}\end{array}$ \\
\hline 183310 & 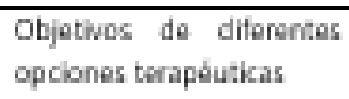 & 1 & 2 & 3 & 4 & $5 \phi \Delta$ \\
\hline 183311 & 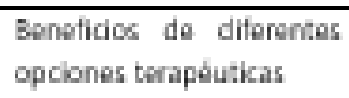 & 10 & 2 & 30 & $4 \Delta$ & 5 \\
\hline 783315 & $\begin{array}{l}\text { Eftector advergos de la } \\
\text { madicacion }\end{array}$ & 1 & 2 & 3 & 40 & $5 \phi \Delta$ \\
\hline 783317 & 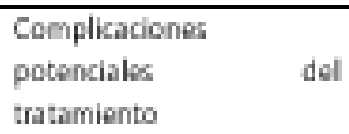 & 1 & 2 & 30 & 4 & $5 \phi \Delta$ \\
\hline 183318 & $\begin{array}{l}\text { Signas y shintomas do } \\
\text { complikadonali } \\
\text { tramianto }\end{array}$ & 1 & 2 & 30 & 4 & $5 \phi \Delta$ \\
\hline 183320 & 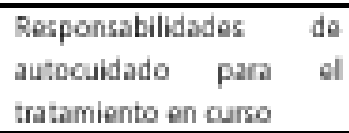 & 1 & 2 & 3 & 40 & $5 \phi \Delta$ \\
\hline 183121 & 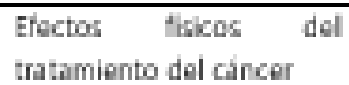 & 1 & 20 & 3 & 4 & $5 \phi \Delta$ \\
\hline 18312 & $\begin{array}{l}\text { Efoctor sobra al astilo do } \\
\text { wia }\end{array}$ & 1 & 20 & 3 & 4 & $5 \phi \Delta$ \\
\hline 783125 & 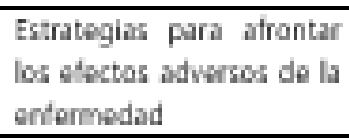 & 10 & 2 & 3 & 4 & $5 \phi \Delta$ \\
\hline 103931 & $\begin{array}{l}\text { Ginpos da apoyo } \\
\text { dtoponbles }\end{array}$ & 10 & 2 & $3 \phi$ & $4 B$ & 5 \\
\hline
\end{tabular}

Fuente: NOC y Elabaración propia cama se encontra

\section{RECOMENDACIONES}

Promover la metodología de la taxonomía NANDA y las taxonomías del NOC, NIC en las diferentes áreas del cuidado de enfermería. Hacer uso de las Guías de Práctica Clínica (GPC) en el personal de enfermería, ya que favorecen a la toma de decisiones y fundamentan los cuidados de enfermería con mayor precisión. objetiva a lograr $\Delta \log r o$

\section{CONFLICTO DE INTERESES}

Los autores declaran no tener conflicto de intereses.

\section{FINANCIAMIENTO}

Este trabajo fue financiado por los autores 


\section{REFERENCIAS BIBLIOGRÁFICAS}

1. Molgo M, Harz I, Andrighetti F, Del Puerto T, Brañes J, González S. Carcinoma espinocelular de la vulva: caso clínico. Rev chil obstet ginecol Revista Chilena Obstétrica Ginecológica [Internet] 2014 [consultado 22 Nov. 2017]; 79(5): 435-438. Disponible en https://scielo.conicyt.cl/scielo. php?script=sci_arttext\&pid=\$0717-75262014000500012

2. Parellada Cl, Gay de Pereyra EA, Bittencourt A. Neoplasia Intraepitelial Vulvar. Medigraphic. [Internet].2011 [consultado 22 Nov. 2017]; 3(4) 15-24. Disponible en http://www. medigraphic.com/pdfs/archivostgi/tgi-2011/tgi114d.pdf

3. Instituto Nacional de Estadística y Geografía. Comunicación social 2016. [Internet]. Estadística a propósito del día mundial contra el cáncer. [consultado 22 Nov. 2017]; Disponible en http://www.diputados.gob.mx/sedia/biblio/usieg/ comunicados/salud.pdf

4. Instituto Mexicano del Seguro Social. 2015. [Internet]. Guía de Referencia Rápida Diagnóstico y tratamiento de Vulvodinia en los Tres Niveles de Atención. México D.F. Centro Nacional de Excelencia Tecnológica en Salud. Catálogo maestro de guías de práctica clínica IMSS 755-15.2015. [consultado 22 Nov. 2017]; Disponible en http://www.cenetec-difusion.com/ CMGPC/IMSS-755-15/RR.pdf

5. Flores I, Patlán R, Reyna H, Solano P, Navarro F, Fernández A. Guía de Práctica Clínica. Diagnóstico y Tratamiento de Cáncer de Vagina en el segundo y tercer Nivel de Atención. [Internet]. México, D.F. Consejo de Salubridad General. 2013. [consultado 22 Nov. 2017]; Disponible en http://www. cenetec-difusion.com/CMGPC/IMSS-590-13/ER.pdf

6. Herdman H, Kamitsuru, S. NANDA International. Diagnósticos Enfermeros 2015-2017 Hispanoamerica 97884-9022-952-1 España Editorial Elsevier 2014.1-475.

7. Gordon M. Manual de diagnósticos de enfermería, 11 ediciones. México, D.F. Ed Mc Graw Hill Interamericana de España. 2007.

8. Bulechelk G, Butcher H, Docheterman J, Wagner Ch. Clasificación de Intervenciones de Enfermería (NIC). 6a ed. Barcelona España Elsevier, 2013 1-634.

9. Moorhed S, Johnson, M, Mass M, Swanson E. Clasificación de Resultados de Enfermería (NOC). $5 a$ ed. Barcelona España: Elesevier; 2013. 1-736. dunab\&page $=$ article\&op $=$ viewArticle\&path\%5B\%5D =2211

10. Chávez WO, Becerra CP, Naranjo AS. Factores que intervienen en el manejo del dolor oncológico: un reto para el profesional de enfermería. Med UNAB [Internet] 2016 [consultado12 Ene. 2018]; 19 (1) 18-24. Disponible en https:// revistas.unab.edu.co/index.php/medunab/article/view/2211

11. Robert V, Álvarez C, Valdivieso F. Psicología: Un modelo de intervención y apoyo social. REV.MED.CLIN.CONDES Revista Médica Clínica Condes[Internet] 2013 [consultado Diciembre. 2017]; 24(4): 677-684. Disponible en https://www. clinicalascondes.cl/Dev_CLC/media/Imagenes/PDF\%20 revista\%20m\%C3\%A9dica/2013/4\%20julio/14_Ps.-Ver\%C2\%A6nica-Robert-M.pdf

12. Información sobre la medicina integrativa y las terapias complementarias 2016 [Internet] Nueva York, N.Y. Sociedad de lucha contra la leucemia y el linfoma; [consultado Diciembre. 2017].1-9 Disponible en https://www.lls.org/ sites/default/files/file_assets/FS8S_Span_Integrative\%20 Medicine\%20Facts_FINAL_Mar2017.pdf

13. Triviño Z, Sanhueza, A. Teorías y modelos relacionados con calidad de vida en cáncer y enfermería. Aquichan [Internet].2005 [consultado 15 Febrero. 2019]; 5(1) 20-31. Disponible en http://aquichan.unisabana.edu.co/index.php/ aquichan/article/view/56/117\%3E.

14. Vega P, González R, Palma Ch, Ahumada E, Mandiola J, Oyarzun C, Rivera S. Develando el significado del proceso de duelo en enfermeras (os) pediátricos (os) que se enfrentan a la muerte de un paciente a causa del cáncer. Aquichan. [Internet] 2013 [consultado12 Ene. 2018] 13(1).8191. Disponible en http://aquichan.unisabana.edu.co/index. php/aquichan/article/view/2400

15. Moreno A, Krikorian A, Palacio C. Malestar emocional, ansiedad y depresión en pacientes oncológicos colombianos y su relación con la competencia percibida Avances en psicología latinoamericana/Bogotá [Colombia]. [Internet] 2015 [consultado12 Ene. 2018] 33(3).517529. Disponible en https://revistas.urosario.edu.co/ xml/799/79940724011/79940724011_visor_jats.pdf 